\title{
Accuracy assessment of a novel optical image guided system for trans-nasal sinus and skull base surgeries
}

\author{
Dr. Yerasimos Kyriakides \\ Department of Otolaryngology - Head and Neck Surgery, Aretaeio Hospital, Nicosia, Cyprus
}

\begin{abstract}
Purpose: Many studies have been conducted on assessment of navigation accuracy on cadaver head using pre-installed CT fiducials. However, there are fewer studies on assessing accuracy on real patient due to its complexity in measuring Target Registration Error (TRE). In this paper we propose a new approach for quantifying navigation accuracy on the real patients. This paper summarizes the results of navigation accuracy of a novel optical image guided navigation system evaluated on over 20 patients.

Method: Commercially available optical navigation system (Navient Image guided navigation system - ClaroNav Kolahi Inc., Toronto, Canada) was used on over 20 patients from 2017 to 2019. Navient employs both landmarks based and trace based registration. Since mounting CT fiducials on the real patients is invasive and impractical, we developed a new approach to measure the distance from the navigation probe to the patient's skin as a new metric to quantify navigation accuracy. The measurement was conducted in dozens of locations including patient's forehead, temples and base of the nose. These anatomical locations were chosen very carefully to represent navigation accuracy in coronal, sagittal and axial directions respectively. Furthermore, our method also eliminates human errors involved in the TRE measurements which is the main source of error in TRE studies.

Results: The average Euclidian distance from skin on 23 patients were $1.47(\mathrm{~mm})$. the standard deviation of the accuracy was $0.28(\mathrm{~mm})$. Low STD demonstrates consistent accuracy during the two year course of the study.

Conclusion: A new noninvasive approach for accuracy assessment of image guided navigation systems on real patient was proposed and validated using a novel optical navigation system. The low STD of navigation accuracy indicates its reliable accuracy.
\end{abstract}

\section{Introduction}

X-ray imaging was discovered by Wilhelm Roentgen in 1895. For the first time, surgeons could see inside a patient without cutting them open. However, these were 2D projections of the anatomy on film. In 1972, Godfrey Hounsfield and Allan Cormack invented a method to acquire and digitally process X-ray projections from many directions to produce a $3 \mathrm{D}$ representation of the patient's anatomy. This new type of device was initially called computerized axial tomography (CAT), and later shortened to CT. Hounsfield and Cormack were later awarded the Nobel Prize for their contributions to medicine and science.

Initially, brain surgeons used $\mathrm{CT}$ images to guide needles into specific locations in the head using a mechanical guide in the form of a large metal "stereotaxic frame", screwed to the patient's skull. In the late 1980s, four different research groups (at Dartmouth, Aachen, Tokyo and Vanderbilt) concurrently and independently developed prototypes that could register a patient's head with its CT images, then dynamically show the tip of a pointer mapped to its corresponding location in those images.

During 1990-94, a Canadian company (ISG Technologies) developed and launched the first surgical navigation product, the Viewing Wand. Shortly after the introduction of the Viewing Wand, other companies introduced similar products, and in the following 5 years, research into the use of surgi- 
cal navigation systems rapidly expanded to other anatomical regions, including sinuses, spine, hips and knees.

Initially, this new technology was called "frameless stereotaxy", implying that its main benefit was the elimination of the need for the cumbersome head frame. However, it quickly became clear that the devices not only improved surgical results by making brain surgery more precise, but also enabled surgery to become much less invasive, reducing patient morbidity and the risks of infections. By the early 2000s, surgical navigation had become standard-ofcare in neurosurgery, and was starting to become increasingly popular in sinus surgery as well.

Studies show that Surgical Navigation Systems would allow more complete dissection, obviating the need for revision surgery. It helps surgeons make more informed decisions and enables better patient outcomes [1]. It has become a valuable aid for many endoscopic sinus surgeries including transphenoidal procedures, maxillary antrostomnies, ethmoidectomies, sphenoidectomies, sphenoid explorations, turbinate resections, frontal sinusotomnies, intranasal procedures, intranasal tumor resections, and ENT related skull base surgery.

Navigation accuracy is, perhaps, the most important feature of all navigation systems. The accuracy is highly dependent on registration methods and the type of tracking system used. The registration method used in most navigation systems is point to point skin landmark registration, surface-based registration or a combination of both. However, there are two different technologies for tracking systems: electromagnetic and optical.

Electromagnetic surgical navigation systems facilitate instruments orientation in anatomic settings such as the paranasal sinuses and the anterior skull base. The surgeon can move the instruments freely without dependence on an uninterrupted line of sight [2-4]. However optical systems are easier to set up and provides wireless tracking. Unlike electromagnetic systems, accuracy of optical systems does not suffer from the interference of ferromagnetic objects within the magnetic transmitter field. As concluded in our study, optical systems provide reliable and consistent navigation accuracy. The average RMS of the optical tracking systems is $0.25 \mathrm{~mm}$ [5], while the average RMS of electromagnetic tracking systems is $1.20 \mathrm{~mm}$ [6].

\section{Principle of operation}

Optical navigation system's guidance function is based on CT image data acquired prior to the procedure, combined with optical measurements of the pose of navigated instruments relative to the patient's head. The Patient Tracker is attached to the patient's forehead to enable tracking of the patient's head, and the image data is loaded by the navigation software. The image data is then spatially registered with the patient's head by point to point registration, followed by trace-based registration. Once the registration process is completed, the system presents the location of a navigated instrument tip overlaid on the image data.

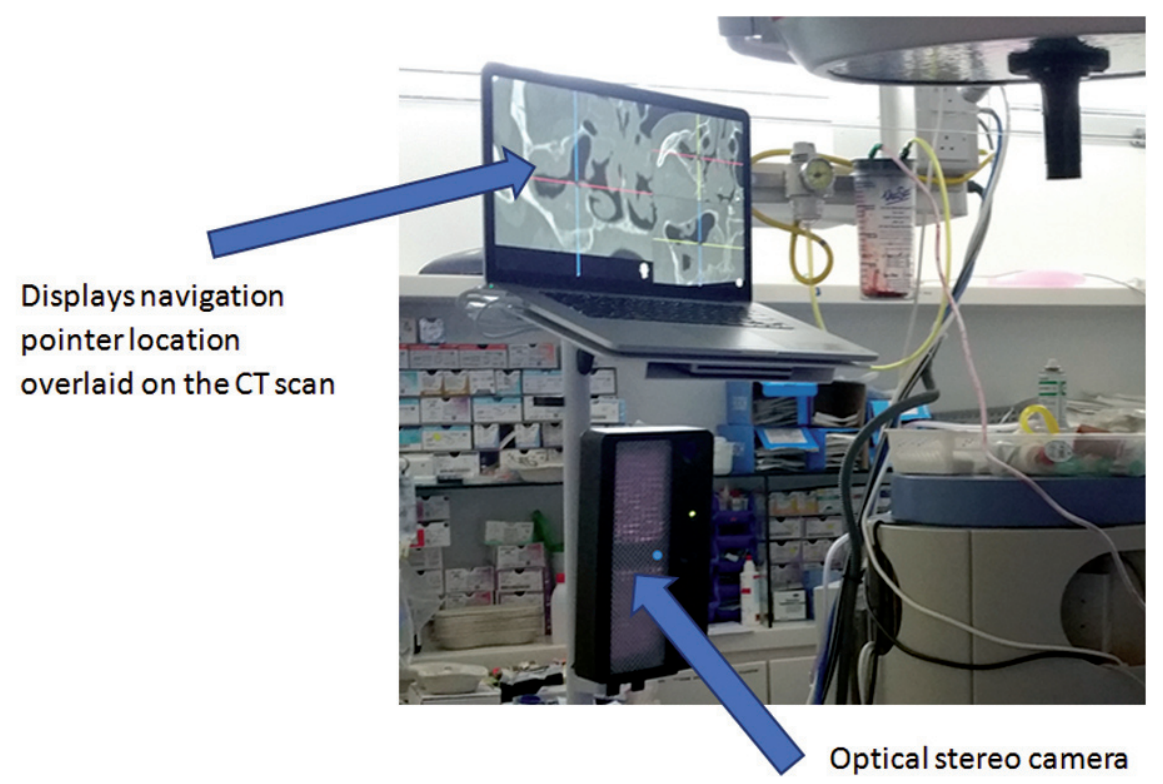


The optical tracking Camera box contains a stereoscopic camera and an infrared (IR) light source, both operating in the near infrared (NIR) light spectrum. The tacking software identifies target marker mounted on the navigation instruments. Software processes the stereoscopic video images to detect and triangulate the positions of the markers. The location and orientation of the tip of each instrument relative to its markers is stored in the marker template and is used in mapping the tip to the CT image.

\section{Set up}

Standard operating room setup for endoscopic trans-nasal and skull base surgery were used. The Navient navigation system was placed next to the endoscope cart, with its screen as close as possible to the endoscope monitor to reduce the head movement when switching the view between the two monitors. A self-adhesive patient tracker was attached to the forehead.

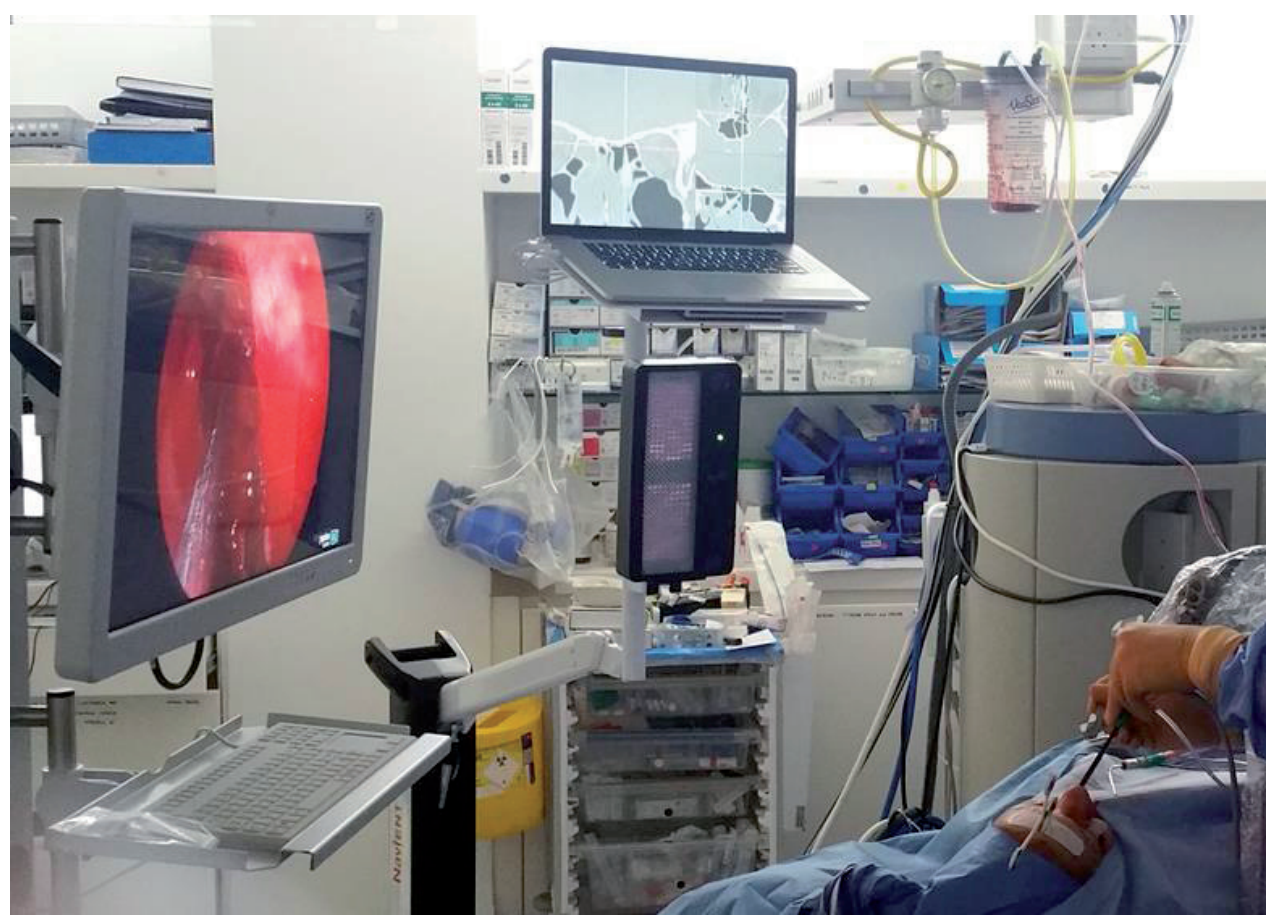

Navigation placed next to endoscope tower and the Patient tracker attached on the forehead

\section{Study design}

Many studies have been conducted on accuracy assessment of navigation systems on cadaver heads, while fewer studies have been conducted to evaluate accuracy on real patient because it is not practical to mount CT fiducials or screws on the patient to measure TRE. We developed a new approach to overcome this obstacle and yet generate a very reliable metric to quantify navigation accuracy.

In this study we measure the distance from the pointer tip to the patient skin. The measurement is done in dozens of points on the patient face, including forehead, temples and base of the nose. These points are carefully chosen to represent coronal, sagittal and axial planes respectfully, and therefore any inaccuracy in any of 3 planes will be detected in our measurements. Furthermore, unlike the conventional TRE measurement approach, which is prone to human error in inaccurately identifying the fiducial and placing the pointer on it, our approach eliminates human error completely.

Over 20 endoscopic sinus and skull base surgeries were performed during the two years course of study. Standard CT scan of the patients with slice thickness of $0.3 \mathrm{~mm}$ to $0.63 \mathrm{~mm}$ were acquired prior to surgery. Navient's point to point landmark-based registration followed by trace-based registration was used to map CT images to patient's head. The distance from navigation probe to patient's skin was measured in dozens of locations including forehead, temples and base of the nose. We took the following measures to minimize unintended errors during the measurement: 
1. A ball tip navigation pointer was used to minimize the inaccuracy due to the tip penetrating into skin.

2. The points were collected from patient face where it has thin skin and bony structure.

3. We paid extra attention not to push the instrument tip into the patient skin

4. The points were collected from the surface area that encapsulate the sinuses and skull base

\section{Data analysis}

Table below displays the average error calculated for each individual patient. It is calculated from the multiple measurements that were taken from patient's forehead, temples and base of the nose.

We also collected CT scan spacing info to investigate the impact of scans resolution on the navigation accuracy.

\begin{tabular}{|c|c|c|c|c|}
\hline Patient no. & Sex / Age & OR Date & CT scan spacing (mm) & $\begin{array}{l}\text { Average distance from } \\
\text { surface }(\mathrm{mm})\end{array}$ \\
\hline 1 & $F / 55$ & $2017-10-27$ & $0.38,0.38,0.45$ & 1.53 \\
\hline 2 & $\mathrm{~F} / 30$ & 2017-11-11 & $0.42,0.42,0.50$ & 0.99 \\
\hline 3 & $\mathrm{M} / 80$ & 2017-11-14 & $0.44,0.44,0.50$ & 1.46 \\
\hline 4 & $\mathrm{M} / 41$ & $2017-11-21$ & $0.44,0.44,0.50$ & 1.18 \\
\hline 5 & $M / 31$ & $2017-11-30$ & $0.34,0.34,0.30$ & 1.03 \\
\hline 6 & $\mathrm{~F} / 44$ & 2017-12-02 & $0.32,0.32,0.50$ & 1.32 \\
\hline 7 & M / 17 & $2017-12-21$ & $0.35,0.35,0.50$ & 1.39 \\
\hline 8 & M / 39 & 2018-01-13 & $0.38,0.38,0.30$ & 1.55 \\
\hline 9 & $F / 38$ & 2018-02-01 & $0.49,0.49,0.49$ & 1.59 \\
\hline 10 & M / 59 & $2018-02-24$ & $0.36,0.36,0.80$ & 1.35 \\
\hline 11 & $M / 39$ & 2018-04-12 & $0.42,0.42,0.50$ & 1.40 \\
\hline 12 & $\mathrm{~F} / 53$ & 2018-04-17 & $0.43,0.43,0.63$ & 1.41 \\
\hline 13 & F / 18 & $2018-05-20$ & $0.35,0.35,0.30$ & 1.49 \\
\hline 14 & F / 22 & 2018-07-05 & $0.35,0.35,0.50$ & 2.14 \\
\hline 15 & $\mathrm{M} / 49$ & $2018-07-28$ & $0.36,0.36,0.50$ & 2.14 \\
\hline 16 & F / 29 & 2018-07-28 & $0.34,0.34,0.50$ & 1.58 \\
\hline 17 & F / 48 & 2018-09-26 & $0.37,0.37,0.50$ & 1.62 \\
\hline 18 & M / 64 & 2018-09-27 & $0.52,0.52,0.50$ & 1.51 \\
\hline 19 & $\mathrm{M} / 50$ & $2018-10-24$ & $0.35,0.35,0.30$ & 1.23 \\
\hline 20 & $M / 33$ & 2018-11-13 & $0.47,0.47,0.63$ & 1.46 \\
\hline 21 & $M / 48$ & 2019-09-06 & $0.27,0.27,0.50$ & 1.29 \\
\hline 22 & $M / 26$ & 2019-11-01 & $0.35,0.35,0.40$ & 1.77 \\
\hline 23 & $M / 34$ & $2019-12-27$ & $0.46,0.46,0.63$ & 1.37 \\
\hline
\end{tabular}

Average value of multiple measurements of distance between the pointer tip to the patient skin for each patient is calculated.

Among the records reported in the table above, patients no. 14 and no. 15 were identified as outliers. Further investigation demonstrated that the scans from these patients were missing the forehead and tip of the nose. It is expected that this will impact Navient's trace-based registration negatively, as the trace algorithm is matching a path traced by the user on the patient's face with a model of patient's face surface generated from the image data. Therefore, we removed these two cases from further analyses.

\section{Results}

The average error on 21 patients was $1.40 \mathrm{~mm}$, with STD of $0.19 \mathrm{~mm}$. the small STD demonstrates reliability of the accuracy across all patients. All patients were scanned with submillimeter slice spacing and we found no significant correlation between the accuracy and the spacing value. 


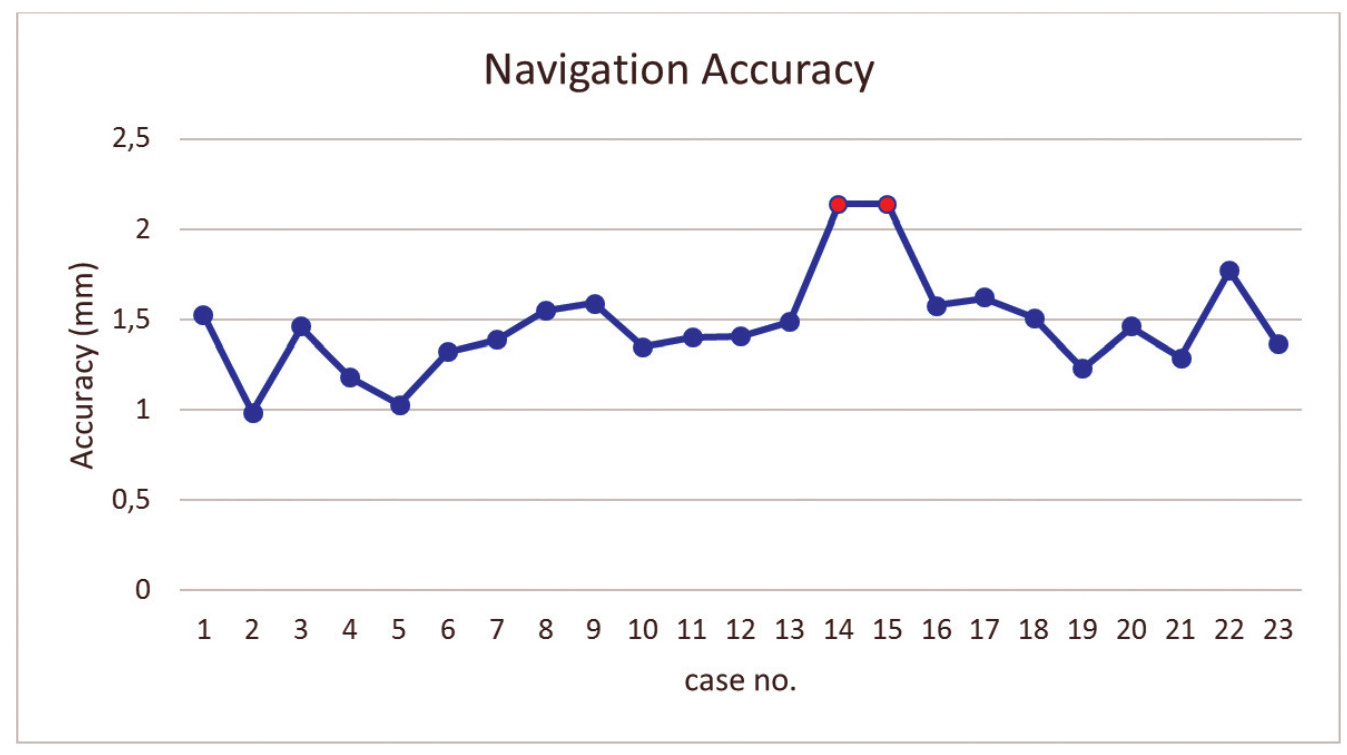

The $\mathrm{x}$ - axis lists the patients, the $\mathrm{y}$ - axis contains the average error of each patient in $\mathrm{mm}$.

\section{Conclusion}

We developed a new approach for accuracy assessment of navigation systems on real patients. The approach is completely non-invasive and based on a measuring the distance from the pointer tip to the patient skin in three different planes. It doesn't require any $\mathrm{CT}$ fiducial and therefore can be conducted on real patients. The study was conducted using Navient optical navigation system on 21 patients and the average error was $1.40 \mathrm{~mm}$.

\section{References}

1. Robert F. Labadie and J. Michael Fitzpatrick (new edition March 2016) Image-Guided Surgery: Fundamentals and Clinical Applications in Otolaryngology. Plural Publishing Inc;

2. Hahn P, Oezdemir S, Komp M, Giannakopoulos A, Kasch R, et al. (2015) Navigation of pedicle screws in the thoracic spine with a new electromagnetic navigation system: a human cadaver study. Biomed Res Int 2015: 183586.

3. Kral F, Puschban EJ, Riechelmann H, Pedross F, Freysinger W (2011) Optical and electromagnetic tracking for navigated surgery of the sinuses and frontal skull base. Rhinology 49: 364-368.

4. Freysinger C (2003) Computer assistance for intraoperative navigation in ENT surgery. Minim Invasive Ther Allied Technol 12: 36-51.

5. https://www.ndigital.com/medical/products/polaris-vega/

6. https://www.ndigital.com/medical/products/aurora/\#specifications-tabletop-field 